\title{
New Naphthoquinone Derivatives from the Ascomycete IBWF79B-90A
}

\author{
Anja Schüffler ${ }^{\mathrm{a}}$, Johannes C. Liermann ${ }^{\mathrm{b}}$, Heinz Kolshorn ${ }^{\mathrm{c}}$, Till Opatz \\ and Heidrun Anke ${ }^{\mathrm{d}, *}$ \\ a University of Kaiserslautern, Paul-Ehrlich-Str. 23, D-67663 Kaiserslautern, Germany \\ b Institute of Organic Chemistry, University of Hamburg, Martin-Luther-King-Platz 6, \\ D-20146 Hamburg, Germany \\ c Institute of Organic Chemistry, University of Mainz, Duesbergweg 10-14, D-55128 \\ Mainz, Germany \\ d Institute of Biotechnology and Drug Research, Erwin-Schrödinger-Str. 56, D-67663 \\ Kaiserslautern, Germany. E-mail: anke@ibwf.de \\ * Author for correspondence and reprint requests \\ Z. Naturforsch. 64c, 25-31 (2009); received September 23/October 24, 2008
}

Bioactivity-guided fractionation of extracts from the fungus IBWF79B-90A resulted in the isolation of three known naphthoquinones, herbarin, dehydroherbarin, and $O$-methylherbarin and the azaanthraquinone scorpinone as well as three structurally related derivatives, $O$-phenethylherbarin and herbaridines A and B. All seven compounds exhibited cytotoxic activities against several cell lines.

Key words: Naphthoquinone, Herbaridine, Cytotoxic Activity

\section{Introduction}

Naphthoquinone metabolites are widespread in nature and show a wide range of biological activities. Closely related structures to those described herein could be found in plants, e.g. psychorubin isolated from Psychotria rubra (Hayashi et al., 1987) as well as nanaomycin A isolated from Streptomyces species (Tanaka et al., 1975). A considerable number of naphthoquinones have also been isolated from fungi. Species of the genus Fusarium produce a wide variety of related metabolites, e.g. fusarubins, which exhibit antimicrobial, cytotoxic (Kurobane et al., 1986), and phytotoxic (Medentsev and Akimenko, 1992) activities. Fusarium species are able to produce azaanthraquinones like bostrycoidin, which was the first isolated natural 2-aza-anthraquinone (Arsenault, 1965); its structure is related to scorpinone isolated here from IBWF79B-90A and previously from a Bisporalike tropical fungus (Miljkovic et al., 2001). The mechanism of the biosynthesis was studied and indicated the existence of a common precursor which is modified through reduction, dehydration or oxidation and leads to the variability of derivatives (Medentsev and Akimenko, 1998).
Seven structurally related naphthoquinones were isolated from the ascomycete IBWF79B-90A, and three of them are new. The known compounds herbarin, dehydroherbarin, and $O$-methylherbarin were first isolated from the fungus Torula herbarum (Kadkol et al., 1971; Narasimhachari and Gopalkrishnan, 1974). Their biological activity is comparable to related, already known compounds like the fusarubins.

\section{Material and Methods}

\section{Producing organism}

The sterile filamentous ascomycete IBWF79B90A could neither be identified by microscopic characteristics nor by ITS sequencing. The ITS sequence determined by us is available through GenBank (accession number EU848215), the fungus is deposited in the culture collection of the Institute of Biotechnology and Drug Research (IBWF e.V.), Kaiserslautern, Germany. For maintenance the fungus was grown on agar slants on YMG agar (4 g/l yeast extract, $10 \mathrm{~g} / \mathrm{l}$ malt extract, $10 \mathrm{~g} / \mathrm{l}$ glucose; the $\mathrm{pH}$ value was adjusted to 5.5 before autoclaving). Solid media contained $2 \%$ of agar. 
Fermentation and isolation of the naphthoquinones

The fungus was grown in YMG medium in a 20-1 fermenter (DCI-Biolafitte, St. Cloud, MN, USA) at $22-24^{\circ} \mathrm{C}$ with agitation $(130 \mathrm{rpm})$ and aeration $(3 \mathrm{l} / \mathrm{min})$. For inoculation a well grown shake culture $(250 \mathrm{ml})$ in the same medium from a 500-ml Erlenmeyer flask was used. During the fermentation, the carbon source was depleted after $11 \mathrm{~d}$. The highest metabolite concentration was reached after $19 \mathrm{~d}$ as judged by analytical HPLC. The fermentation was stopped and the culture fluid was separated from the mycelia through filtration. The culture fluid (171) was extracted twice with EtOAc and the combined extracts were dried. This crude extract $(1.5 \mathrm{~g})$ was applied onto silica gel (Merck 60, 0.063-0.2 mm, $75 \mathrm{~g}$ ). Elution with cyclohexane/EtOAc (3:1) yielded intermediate I $(56.8 \mathrm{mg})$, with cyclohexane/EtOAc (1:1) intermediate II $(293.8 \mathrm{mg})$, and with EtOAc intermediate III $(468.0 \mathrm{mg})$. The purification of the compounds was achieved by preparative HPLC (Merck, LiChroSorb RP18, $7 \mu \mathrm{m}, 250 \times$ $25 \mathrm{~mm}$ ) with $\mathrm{MeCN} / \mathrm{H}_{2} \mathrm{O}$ gradients. Intermediate I furnished $0.6 \mathrm{mg}$ herbarin (4) (RT $31.4 \mathrm{~min}$ ) and $0.8 \mathrm{mg} O$-phenethylherbarin (1) (RT $51.9 \mathrm{~min}$ ) with a $\mathrm{MeCN} / \mathrm{H}_{2} \mathrm{O}$ gradient $(20 \%$ to $70 \% \mathrm{MeCN}$ in $70 \mathrm{~min}$; flow, $25 \mathrm{ml} / \mathrm{min}$ ). Dehydroherbarin (5) $(4.7 \mathrm{mg}$; RT $39.5 \mathrm{~min}$; in $50 \mathrm{~min}$ from $35 \%$ to $70 \% \mathrm{MeCN}$; flow, $20 \mathrm{ml} / \mathrm{min}$ ) was isolated from intermediate II. Intermediate III was separated with a $\mathrm{MeCN}$ gradient $(10 \%$ to $50 \%$ in $50 \mathrm{~min}$; flow, $25 \mathrm{ml} / \mathrm{min}$ ) and yielded $O$-methylherbarin (6) (5.3 mg; RT $29.0 \mathrm{~min}$ ), scorpinone (7) $(2.5 \mathrm{mg}$; RT $31.2 \mathrm{~min}$ ) and a mixture of herbaridines A (2) and B (3) (19.0 mg). This mixture was separated by semipreparative HPLC (Merck, LiChroSpher $100, \mathrm{RP} 18,5 \mu \mathrm{m}, 125 \times 5 \mathrm{~mm} ; 1 \%$ to $100 \% \mathrm{MeCN}$ in $20 \mathrm{~min}$; flow, $1 \mathrm{ml} / \mathrm{min}$ ) and yielded herbaridine A (2) (1.9 mg; RT $8.7 \mathrm{~min})$ and herbaridine B (3) (13.3 mg; RT $11.8 \mathrm{~min}$ ).

\section{Spectroscopic characterization}

Melting points were determined with a Dr. Tottoli apparatus (Büchi) and are uncorrected. Optical rotations were measured with a Krüss P8000 polarimeter at $589 \mathrm{~nm}$. UV and IR spectra were measured with a Perkin-Elmer Lambda-16 spectrophotometer and a Bruker IFS48 FTIR spectrometer, respectively. NMR spectra were record- ed in $\mathrm{CDCl}_{3}$ with a Bruker Avance II (400 MHz) or DRX-500 (500 MHz) spectrometer, the chemical shifts were referenced to the residual solvent signal $\left(\mathrm{CDCl}_{3} \delta_{\mathrm{H}}=7.26 \mathrm{ppm}, \delta_{\mathrm{C}}=77.16 \mathrm{ppm}\right.$; Gottlieb et al., 1997). APCIMS spectra were measured with a Hewlett Packard MSD1100 instrument. FAB mass spectra were measured with a Vacuum Generators VG70S spectrometer (Xe-FAB ionization) using $m$-nitrobenzyl alcohol or glycerol as matrix. HRFABMS data were determined using PEG 300 or 600 as the reference.

\section{Physiochemical properties}

O-Phenethylherbarin (1): Yellow solid, m.p. $143-144^{\circ} \mathrm{C} .-[a]_{\mathrm{D}}^{25}+66.0^{\circ}\left(c 0.043, \mathrm{CDCl}_{3}\right) .-\mathrm{UV}$ $(\mathrm{MeOH}): \lambda_{\max }(\log \varepsilon)=214$ (4.51), 265 (4.11), 411 (3.48) nm; $\lambda_{\text {min }}(\log \varepsilon)=230(3.90), 318$ (2.99) nm. - IR (KBr): $v=3436,2939,1657,1595,1456,1330$, $1278,1213,1158,1101,1049,701 \mathrm{~cm}^{-1} .-{ }^{1} \mathrm{H}$ and ${ }^{13} \mathrm{C}$ NMR: see Tables I and II. - FABMS: $m / z(\%)=$ $245.1 \quad\left[\mathrm{M}-\mathrm{CH}_{3} \mathrm{COO}\left(\mathrm{CH}_{2}\right)_{2} \mathrm{Ph}+\mathrm{H}\right]^{+} \quad$ (31), 287.1 $\left[\mathrm{M}-\mathrm{O}\left(\mathrm{CH}_{2}\right)_{2} \mathrm{Ph}\right]^{+}(22), 409.2[\mathrm{M}+\mathrm{H}]^{+}(14) .-\mathrm{HR}-$ FABMS: $m / z=409.1656 ;\left[\mathrm{C}_{24} \mathrm{H}_{24} \mathrm{O}_{6}+\mathrm{H}\right]^{+}$requires $m / z=409.1646$.

Table I. ${ }^{1} \mathrm{H}$ NMR data $\left(400 \mathrm{MHz}, \mathrm{CDCl}_{3}\right)$ of $\mathbf{1}, \mathbf{2 a}$, and 3. Coupling constants $(J)$ are given in $\mathrm{Hz}$.

\begin{tabular}{|c|c|c|c|}
\hline $\mathrm{H}$ & 1 & $2 \mathbf{a}$ & 3 \\
\hline \multirow[t]{4}{*}{1} & 4.61; ddd; & 4.24; dd; & 4.10 ; dd; \\
\hline & $18.8,2.6,0.9$ & $11.9,10.6$ & $11.9,4.7$ \\
\hline & 4.14; ddd; & 4.13; dd; & 3.88; dd; \\
\hline & $18.8,4.2,3.1$ & $11.9,4.7$ & $11.9,10.7$ \\
\hline \multirow[t]{4}{*}{4} & 2.82; ddd; & 2.37; dd; & 2.33; dd; \\
\hline & $18.6,3.1,0.9$ & $13.7,3.8$ & $13.8,3.8$ \\
\hline & 2.46; ddd; & $1.72 ; \mathrm{dd}$ & $1.72 ; \mathrm{dd} ;$ \\
\hline & $18.6,4.2,2.6$ & $13.7,11.7$ & $13.8,11.6$ \\
\hline $4 a$ & - & $\begin{array}{c}\text { 3.38; ddd; } \\
13.2,11.7,3.8\end{array}$ & $\begin{array}{l}\text { 3.37; ddd; } \\
13.2,11.6,3.8\end{array}$ \\
\hline 6 & $7.27 ; \mathrm{d} ; 2.5$ & $7.14 ; \mathrm{d} ; 2.5$ & $7.13 ; \mathrm{d} ; 2.4$ \\
\hline 8 & $6.72 ; \mathrm{d} ; 2.5$ & $6.72 ; \mathrm{d} ; 2.5$ & $6.71 ; \mathrm{d} ; 2.4$ \\
\hline $10 \mathrm{a}$ & - & $\begin{array}{c}2.92 ; \mathrm{ddd} ; \\
13.2,10.6,4.7\end{array}$ & $\begin{array}{l}\text { 2.91; ddd; } \\
13.2,10.7,4.7\end{array}$ \\
\hline $3-\mathrm{CH}_{3}$ & $1.48 ; \mathrm{s}$ & $1.51 ; \mathrm{s}$ & $1.39 ; \mathrm{s}$ \\
\hline $7-\mathrm{OCH}_{3}$ & $3.97 ; \mathrm{s}$ & $3.93 ; \mathrm{s}$ & $3.92 ; \mathrm{s}$ \\
\hline $9-\mathrm{OCH}_{3}$ & $3.95 ; \mathrm{s}$ & $3.92 ; \mathrm{s}$ & $3.91 ; \mathrm{s}$ \\
\hline $1^{\prime}$ & $3.69 ; \mathrm{m}$ & - & $3.23 ; \mathrm{s}$ \\
\hline $2^{\prime}$ & $2.79 ; \mathrm{m}$ & - & - \\
\hline $2 " / 6 "$ & $7.16 ; \mathrm{m}$ & - & - \\
\hline 3"/5" & $7.20 ; \mathrm{m}$ & - & - \\
\hline $4 "$ & $7.16 ; \mathrm{m}$ & - & - \\
\hline $3-\mathrm{OH}$ & - & $\sim 2.0$ & - \\
\hline
\end{tabular}


Table II. ${ }^{13} \mathrm{C}$ NMR data $\left(101 \mathrm{MHz}, \mathrm{CDCl}_{3}\right)$ of $\mathbf{1}, \mathbf{2 a}$, and 3. Multiplicities were determined indirectly by DEPT135 and HSQC.

\begin{tabular}{lccc}
\hline $\mathrm{C}$ & $\mathbf{1}$ & $\mathbf{2 a}$ & $\mathbf{3}$ \\
\hline 1 & $58.67 ; \mathrm{t}$ & $60.29 ; \mathrm{t}$ & $60.27 ; \mathrm{t}$ \\
3 & $97.12 ; \mathrm{s}$ & $95.47 ; \mathrm{s}$ & $97.79 ; \mathrm{s}$ \\
4 & $32.75 ; \mathrm{t}$ & $34.70 ; \mathrm{t}$ & $35.79 ; \mathrm{t}$ \\
$4 \mathrm{a}$ & $136.81 ; \mathrm{s}$ & $45.15 ; \mathrm{d}$ & $45.02 ; \mathrm{d}$ \\
5 & $184.04 ; \mathrm{s}$ & $197.23 ; \mathrm{s}$ & $197.40 ; \mathrm{s}$ \\
$5 \mathrm{a}$ & $136.13 ; \mathrm{s}$ & $139.00 ; \mathrm{s}$ & $139.00 ; \mathrm{s}$ \\
6 & $103.53 ; \mathrm{d}$ & $102.08 ; \mathrm{d}$ & $102.03 ; \mathrm{d}$ \\
7 & $162.04 ; \mathrm{s}$ & $164.79 ; \mathrm{s}$ & $161.29 ; \mathrm{s}$ \\
8 & $104.13 ; \mathrm{d}$ & $104.84 ; \mathrm{d}$ & $104.92 ; \mathrm{d}$ \\
9 & $164.72 ; \mathrm{s}$ & $161.36 ; \mathrm{s}$ & $164.74 ; \mathrm{s}$ \\
$9 \mathrm{a}$ & $114.25 ; \mathrm{s}$ & $118.11 ; \mathrm{s}$ & $118.13 ; \mathrm{s}$ \\
10 & $181.63 ; \mathrm{s}$ & $194.01 ; \mathrm{s}$ & $194.25 ; \mathrm{s}$ \\
$10 \mathrm{a}$ & $143.02 ; \mathrm{s}$ & $48.86 ; \mathrm{d}$ & $48.71 ; \mathrm{d}$ \\
$3-\mathrm{CH}_{3}$ & - & $30.27 ; \mathrm{q}$ & $23.39 ; \mathrm{q}$ \\
$7-\mathrm{OCH}_{3}$ & $56.55 ; \mathrm{q}$ & $56.11 ; \mathrm{q}$ & - \\
9-OCH & $56.11 ; \mathrm{q}$ & $56.56 ; \mathrm{q}$ & - \\
$1^{\prime}$ & $62.50 ; \mathrm{t}$ & - & $48.08 ; \mathrm{q}$ \\
$2^{\prime}$ & $36.66 ; \mathrm{t}$ & - & - \\
1" & $138.93 ; \mathrm{s}$ & - & - \\
$2^{\prime \prime} / 6 "$ & $129.13 ; \mathrm{d}$ & - & - \\
$3 " / 5 "$ & $128.42 ; \mathrm{d}$ & - & - \\
4" & $126.51 ; \mathrm{d}$ & - & \\
\hline
\end{tabular}

Herbaridine A (2):Yellow solid, m.p. $120-121^{\circ} \mathrm{C}$. $-[a]_{\mathrm{D}}^{25}+45.8^{\circ}\left(c\right.$ 0.041, $\left.\mathrm{CDCl}_{3}\right) .-\mathrm{UV}(\mathrm{MeOH}):$ $\lambda_{\max }(\log \varepsilon)=235$ (4.36), 252 (4.26), 279 (3.96), 337 (3.83) nm; $\lambda_{\min }(\log \varepsilon)=245$ (4.23), 270 (3.93), 306 (3.46) nm. - IR (KBr): $v=3446,1682,1596,1457$, $1321,1208,1160,1053,1016 \mathrm{~cm}^{-1} \cdot-{ }^{1} \mathrm{H}$ and ${ }^{13} \mathrm{C}$ NMR of 2a: see Tables I and II; of open-chain ketone 2b: see Table III. - FABMS: $m / z(\%)=289.1$ $[\mathrm{M}-\mathrm{OH}]^{+}(80), 307.1[\mathrm{M}+\mathrm{H}]^{+}(53) .-$ HRFABMS: $m / z=289.1069 ;\left[\mathrm{C}_{16} \mathrm{H}_{18} \mathrm{O}_{6}-\mathrm{OH}\right]^{+}$requires $\mathrm{m} / z=$ 289.1071 .

Herbaridine B (3): Yellow solid, m.p. $153-154{ }^{\circ} \mathrm{C}$. $-[a]_{\mathrm{D}}^{25}+110.7^{\circ}\left(c 0.058, \mathrm{CDCl}_{3}\right)-\mathrm{UV}(\mathrm{MeOH}):$ $\lambda_{\max }(\log \varepsilon)=235$ (4.34), 252 (4.24), 279 (3.94), 338 (3.81) $\mathrm{nm} ; \lambda_{\min }(\log \varepsilon)=245$ (4.21), 270 (3.92), 306 (3.44) nm. - IR (KBr): $v=2939,1683,1596,1457$, $1324,1254,1222,1160,1046,825 \mathrm{~cm}^{-1} .-{ }^{1} \mathrm{H}$ and ${ }^{13} \mathrm{C}$ NMR: see Tables I and II: - FABMS: $m / z(\%)=$ $289.1\left[\mathrm{M}-\mathrm{OCH}_{3}\right]^{+}(54), 321.1[\mathrm{M}+\mathrm{H}]^{+}$(58). - HRFABMS: $m / z=321.1344,\left[\mathrm{C}_{17} \mathrm{H}_{20} \mathrm{O}_{6}+\mathrm{H}\right]^{+}$requires $m / z=321.1333$.

Herbarin (4): Yellow solid, m.p. $205-207^{\circ} \mathrm{C}$ (Lit. 192-193 ${ }^{\circ} \mathrm{C}$; Kadkol et al., 1971). - UV (MeOH): $\lambda_{\max }(\log \varepsilon)=212$ (4.32), 263 (3.99), 404 (3.35) nm; $\lambda_{\text {min }}(\log \varepsilon)=227$ (3.70), $314(2.62)$ nm. - IR (KBr):
Table III. ${ }^{1} \mathrm{H}(400 \mathrm{MHz})$ and ${ }^{13} \mathrm{C}$ NMR data $(101 \mathrm{MHz}$, $\mathrm{CDCl}_{3}$ ) of $\mathbf{2 b}$. Coupling constants $(J)$ are given in $\mathrm{Hz}$. Multiplicities were determined indirectly by DEPT-135.

\begin{tabular}{lcc}
\hline Position & $\delta_{\mathrm{H}}$ & $\delta_{\mathrm{C}}$ \\
\hline 1 & - & $194.01 ; \mathrm{s}$ \\
2 & $3.21 ; \mathrm{ddd} ; 12.5,4.7,2.4$ & $53.07 ; \mathrm{d}$ \\
3 & $3.48 ; \mathrm{ddd} ; 12.5,5.5,4.3$ & $45.24 ; \mathrm{d}$ \\
4 & - & $196.82 ; \mathrm{s}$ \\
$4 \mathrm{a}$ & - & $138.91 ; \mathrm{s}$ \\
5 & $7.15 ; \mathrm{d} ; 2.4$ & $102.18 ; \mathrm{d}$ \\
6 & - & $165.04 ; \mathrm{s}$ \\
7 & $6.73 ; \mathrm{d} ; 2.4$ & $105.02 ; \mathrm{d}$ \\
8 & - & $161.17 ; \mathrm{s}$ \\
$8 \mathrm{a}$ & - & $118.34 ; \mathrm{s}$ \\
$6-\mathrm{OCH}_{3}$ & $3.92 ; \mathrm{s}$ & $56.11 ; \mathrm{q}$ \\
$8-\mathrm{OCH}_{3}$ & $3.94 ; \mathrm{s}$ & $56.02 ; \mathrm{q}$ \\
1' & $4.23,3.67 ; \mathrm{m}$ & $59.95 ; \mathrm{t}$ \\
1" & $3.05 ; \mathrm{dd} ; 18.0,4.3$ & $40.47 ; \mathrm{t}$ \\
& $3.00 ; \mathrm{dd} ; 18.0,5.5$ & \\
$2 "$ & - & $206.56 ; \mathrm{s}$ \\
$3 "$ & $2.28 ; \mathrm{s}$ & $30.49 ; \mathrm{q}$ \\
\hline
\end{tabular}

$v=3378,2954,1656,1639,1598,1561,1469,1324$, 1282, 1219, 1166, 1095, 1069, 1039, 978, 939, 847, $814,729 \mathrm{~cm}^{-1} .-{ }^{1} \mathrm{H}$ NMR (500 MHz, $\left.\mathrm{CDCl}_{3}\right): \delta=$ $7.24(\mathrm{~d}, J=2.4 \mathrm{~Hz}, 1 \mathrm{H}, \mathrm{H}-6), 6.70(\mathrm{~d}, J=2.4 \mathrm{~Hz}$, $1 \mathrm{H}, \mathrm{H}-8), 4.74$ (ddd, $J=18.9,2.7,1.2 \mathrm{~Hz}, 1 \mathrm{H}, \mathrm{H}_{\mathrm{a}}-$ 1), 4.68 (ddd, $\left.J=18.9,3.9,3.0 \mathrm{~Hz}, 1 \mathrm{H}, \mathrm{H}_{\mathrm{b}}-1\right), 3.95$ (s, 3H, OMe), 3.94 (s, 3H, OMe), 2.83 (ddd, $J=$ 18.7, 3.0, $\left.1.2 \mathrm{~Hz}, 1 \mathrm{H}, \mathrm{H}_{\mathrm{a}}-4\right), 2.54$ (ddd, $J=18.7,3.9$, $\left.2.7 \mathrm{~Hz}, 1 \mathrm{H}, \mathrm{H}_{\mathrm{b}}-4\right), 2.30$ (s, $\left.1 \mathrm{H}, \mathrm{OH}-3\right), 1.61$ (s, 3H, $\mathrm{CH}_{3}-3$ ) (Paranagama et al., 2007). - APCIMS pos.: $m / z(\%)=287.1[\mathrm{M}-\mathrm{OH}]^{-}(100), 305.1[\mathrm{M}+\mathrm{H}]^{+}$ (56); APCIMS neg.: $m / z(\%)=286.0\left[\mathrm{M}-\mathrm{H}_{2} \mathrm{O}\right]^{-}$ (52), $304.1[\mathrm{M}]^{-}(100)$.

Dehydroherbarin (5): Crimson solid, m.p. $192-193{ }^{\circ} \mathrm{C}$ (Lit. $189-190{ }^{\circ} \mathrm{C}$; Kadkol et al., 1971). - UV (MeOH): $\lambda_{\max }(\log \varepsilon)=215(4.47)$, 249 (4.11), 277 (4.15), 332 (3.68), 390 (3.49), 483 (3.61) $\mathrm{nm} ; \lambda_{\min }(\log \varepsilon)=234$ (4.07), 258 (4.08), 315 (3.60), 369 (3.45), 427 (3.42) nm. - IR (KBr): $v=1657,1625,1589,1467,1390,1321,1291,1249$, 1223, 1202, 1155, 1068, 1042, 964, 847, $748 \mathrm{~cm}^{-1}$. $-{ }^{1} \mathrm{H}$ NMR $\left(500 \mathrm{MHz}, \mathrm{CDCl}_{3}\right): \delta=7.26(\mathrm{~d}, J=$ $2.5 \mathrm{~Hz}, 1 \mathrm{H}, \mathrm{H}-6), 6.71(\mathrm{~d}, J=2.5 \mathrm{~Hz}, 1 \mathrm{H}, \mathrm{H}-8)$, $5.84(\mathrm{q}, J=0.8 \mathrm{~Hz}, 1 \mathrm{H}, \mathrm{H}-4), 5.12\left(\mathrm{~s}, 2 \mathrm{H}, \mathrm{CH}_{2}\right)$, $3.95(\mathrm{~s}, 3 \mathrm{H}, \mathrm{OMe}), 3.94(\mathrm{~s}, 3 \mathrm{H}, \mathrm{OMe}), 2.00$ (d, $J=$ $0.8 \mathrm{~Hz}, 3 \mathrm{H}, \mathrm{CH}_{3}-3$ ) (Kesteleyn and De Kimpe, 2000). - APCIMS pos.: $\mathrm{m} / z(\%)=287.2[\mathrm{M}+\mathrm{H}]^{+}$ (100); APCIMS neg.: $\mathrm{m} / z(\%)=271.0\left[\mathrm{M}-\mathrm{CH}_{3}\right]^{-}$ (13), $286.0[\mathrm{M}]^{-}(25)$. 
O-Methylherbarin (6): Yellow solid, m.p. 183-184 ${ }^{\circ} \mathrm{C}$ (Lit. $188-190{ }^{\circ} \mathrm{C}$; Narasimhachari and Gopalkrishnan, 1974). $-[a]_{\mathrm{D}}^{25}+59.5^{\circ}(c 0.033$, $\left.\mathrm{CDCl}_{3}\right)$. - UV $(\mathrm{MeOH}): \lambda_{\text {max }}(\log \varepsilon)=212(4.33)$, 262 (4.01), $406(3.32) \mathrm{nm} ; \lambda_{\min }(\log \varepsilon)=230(3.83)$, 316 (2.92) nm. - IR (KBr): $v=3414,2940,1658$, $1595,1565,1465,1426,1331,1277$ 1213, 1184, 1161, 1100, 1052, 941, 851, $832 \mathrm{~cm}^{-1} .-{ }^{1} \mathrm{H}$ NMR $\left(500 \mathrm{MHz}, \mathrm{CDCl}_{3}\right): \delta=7.25(\mathrm{~d}, J=2.4 \mathrm{~Hz}, 1 \mathrm{H}$, $\mathrm{H}-6), 6.58$ (d, $J=2.4 \mathrm{~Hz}, 1 \mathrm{H}, \mathrm{H}-8), 4.71$ (ddd, $J=$ $18.8,2.6,0.8 \mathrm{~Hz}, 1 \mathrm{H}, \mathrm{H}_{\mathrm{a}}-1$ ), 4.45 (ddd, $J=18.8$, $\left.4.2,3.3 \mathrm{~Hz}, 1 \mathrm{H}, \mathrm{H}_{\mathrm{b}}-1\right), 3.95$ (s, $\left.3 \mathrm{H}, \mathrm{OMe}\right), 3.94$ (s, $3 \mathrm{H}, \mathrm{OMe}$ ), 3.29 (s, $\left.3 \mathrm{H}, \mathrm{OCH}_{3}-3\right), 2.80$ (ddd, $J=$ $\left.18.7,3.3,0.8 \mathrm{~Hz}, 1 \mathrm{H}, \mathrm{H}_{\mathrm{a}}-4\right), 2.56$ (ddd, $J=18.7,4.2$, $\left.2.6 \mathrm{~Hz}, 1 \mathrm{H}, \mathrm{H}_{\mathrm{b}}-4\right), 1.51\left(\mathrm{~s}, 3 \mathrm{H}, \mathrm{CH}_{3}-3\right)$ (Narasimhachari and Gopalkrishnan, 1974). - APCIMS pos.: $\mathrm{m} / z \quad(\%)=245.1\left[\mathrm{M}-\mathrm{CH}_{3} \mathrm{COOCH}_{3}+\mathrm{H}\right]^{+}$ (58), $287.1\left[\mathrm{M}-\mathrm{OCH}_{3}\right]^{+}$(100), $319.1[\mathrm{M}+\mathrm{H}]^{+}$(20); APCIMS neg.: $m / z(\%)=318.1[\mathrm{M}]^{-}(60)$.

Scorpinone (7): Yellow solid, m.p. $213-217^{\circ} \mathrm{C}$ (Lit. $214-215^{\circ} \mathrm{C}$; Cameron et al., 1980). - UV $(\mathrm{MeOH}): \lambda_{\max }(\log \varepsilon)=233$ (4.43), 277 (4.13), $395(3.65) \mathrm{nm} ; \lambda_{\min }(\log \varepsilon)=253$ (4.06), 348 (3.31) nm. - IR (KBr): $v=3415,1660,1598,1451,1394$, 1330, 1294, 1221, 1169, 1128, 1059, 1017, 949, 853, $723 \mathrm{~cm}^{-1} .-{ }^{1} \mathrm{H}$ NMR $\left(500 \mathrm{MHz}, \mathrm{CDCl}_{3}\right): \delta=9.41$ (s, 1H, H-1), $7.81(\mathrm{~s}, 1 \mathrm{H}, \mathrm{H}-4), 7.43(\mathrm{~d}, J=2.4 \mathrm{~Hz}$, $1 \mathrm{H}, \mathrm{H}-6), 6.84$ (d, $J=2.4 \mathrm{~Hz}, 1 \mathrm{H}, \mathrm{H}-8), 4.02$ (s, $3 \mathrm{H}, \mathrm{OMe}$ ), 3.99 (s, 3H, OMe), 2.74 (s, 3H, $\left.\mathrm{CH}_{3}-3\right)$ (Kesteleyn and De Kimpe, 2000). - APCIMS pos.: $\mathrm{m} / \mathrm{z}(\%)=284.1[\mathrm{M}+\mathrm{H}]^{+}(100)$; APCIMS neg.: $\mathrm{m} / z$ $(\%)=268.0\left[\mathrm{M}-\mathrm{CH}_{3}\right]^{-}(10), 283.0[\mathrm{M}]^{-}(14)$.

\section{Biological assays}

The minimal inhibitory concentrations (MICs) against bacteria and fungi were determined as described previously (Anke et al., 1989).

Cytotoxicity was assayed as described previously (Schoettler et al., 2006). The cell lines Jurkat (ATCC TIB 152), Colo-320 (DSMZ ACC 144) and L-1210 (DSMZ ACC 123) were grown in RPMI 1640 medium (Invitrogen). Neuro-2A (DSMZ ACC 148) was grown in DMEM medium (Invitrogen). All media were supplemented with $10 \%$ heat-inactivated fetal calf serum (Invitrogen), $65 \mu \mathrm{g} / \mathrm{ml}$ of penicillin $\mathrm{G}$ and $100 \mu \mathrm{g} / \mathrm{ml}$ of streptomycin sulfate.

\section{Results and Discussion}

\section{Isolation and structures}

The naphthoquinones (Fig. 1) were isolated by cytotoxicity-guided fractionation using Jurkat cells for the biological assay. The UV data implied that they are structurally related. $O$-Phenethylherbarin (1) has a nominal mass of 408.2, and HRMS measurements gave an elemental composition of $\mathrm{C}_{24} \mathrm{H}_{24} \mathrm{O}_{6}$, requiring 13 unsaturations. NMR spectra showed the presence of a benzene ring with two meta-coupled aromatic protons attached to carbon atoms resonating at high field. HMBC correlations revealed an 1,3-dimethoxybenzene partial structure. The proton at 7.27 showed an HMBC correlation to one of two carbonyl groups, which should be located in ortho-position. The<smiles>COc1cc(OC)c2c(c1)C(=O)C1=C(C2)C(=O)C2COC(C)(OCCc3ccccc3)OC12</smiles><smiles>[R]O[C@]1(C)C[C@H]2C(=O)c3cc(OC)cc(OC)c3C(=O)[C@H]2CO1</smiles>

$\mathrm{R}=\mathrm{H}$ : Herbaridine $\mathrm{A}(2)$ $\mathrm{R}=\mathrm{Me}$ : Herbaridine $\mathrm{B}(\mathbf{3})$

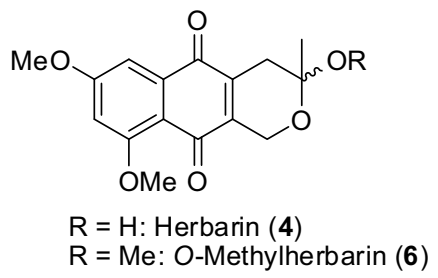<smiles>COc1cc(OC)c2c(c1)C(=O)C1=C(C=C(C)OC1)C2=O</smiles>

Dehydroherbarin (5)<smiles>COc1cc(OC)c2c(c1)C(=O)c1cc(C)ncc1C2=O</smiles>

Fig. 1. Chemical structures of the isolated compounds. 

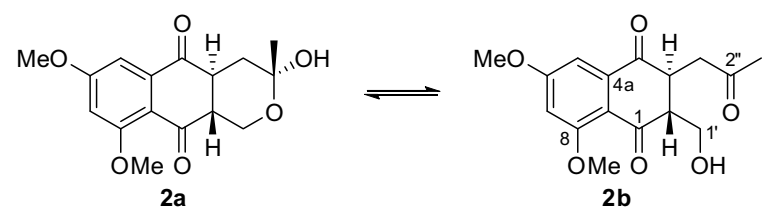

Fig. 2. Ring-opening equilibrium between hemiacetal $2 \mathbf{a}$ and ketone $\mathbf{2 b}$.

same carbonyl group showed HMBC correlations to two methylene protons which exhibited homoallylic ${ }^{5} J$ coupling to the protons of another methylene group over a double bond formed by two quaternary carbon atoms. The latter methylene group gave $\mathrm{HMBC}$ correlations to the other carbonyl group, establishing the structure of a 5,7-dimethoxy-1,4-naphthoquinone. The two methylene groups are part of an anellated dihydropyran unit, forming the 2-phenylethyl acetal of the known pyranonaphthoquinone herbarin (4), and NMR data were well consistent with those reported for 4 (Paranagama et al., 2007) which also exhibited homoallylic ${ }^{5} \mathrm{~J}$ couplings.

Herbaridine A (2) has a nominal mass of 306.1, and HRMS measurements gave an elemental composition of $\mathrm{C}_{16} \mathrm{H}_{18} \mathrm{O}_{6}$, requiring 8 unsaturations. NMR analysis indicated the presence of a similar naphthoquinone scaffold, although no ${ }^{5} \mathrm{~J}$ coupling was observed. All methylene protons exhibited dd multiplicity, and the double bond connecting the carbonyl groups was no longer present. Instead, two vicinal methine carbon atoms were found, and each of the corresponding protons showed coupling with the respective adjacent methylene group. As only a weak NOESY contact but a large ${ }^{3} J$ coupling constant of 13.2 $\mathrm{Hz}$ was observed, both protons should occupy axial positions in the trans-anellated tetrahydropyran ring. The resulting structure is that of a cyclic hemiacetal and almost identical to that of 4, apart from the absence of the central double bond. In the tetrahydropyran ring, the axial protons of the methylene groups could be identified by their upfield chemical shifts and the large axial-axial couplings to the adjacent methine protons. The protons of the methyl group bound to the hemiacetal carbon atom showed nearly equipotent NOESY correlations to both protons of the neighbouring methylene group, so it should be located equatorially. The NOESY spectrum also exhibited exchange with a minor component which was identified as the ring-opened ketone 2b (Fig. 2). Although interconversion of $\mathbf{2 a}$ and $\mathbf{2 b}$ was fast enough to be detected in the NOESY experiment, rendering the anomeric centre permanently labile, the stereoelectronically disfavoured epimer with an axial methyl group was not found in the NMR spectra.

Herbaridine B (3) has a nominal mass of 320.1, an elemental composition of $\mathrm{C}_{17} \mathrm{H}_{20} \mathrm{O}_{6}$ according to HRMS measurements and thus 8 unsaturations. NMR analysis quickly revealed it to be the methyl acetal of herbaridine A (2). Again, the vicinal protons at positions $4 \mathrm{a}$ and $10 \mathrm{a}$ were found in trans-axial position by evaluation of coupling constants and NOESY data. The same holds true for the configuration of the acetal carbon atom which carries the electron withdrawing substituent in axial position, since characteristic NOE contacts were found between the methyl group and the methylene protons at position 4. Due to the acetal structure, ring opening was not observed for $\mathbf{3}$.

\section{Biological properties}

The cytotoxic activities of the compounds are modest. The $\mathrm{IC}_{50}$ values are listed in Table IV. No data on the cytotoxicity of the known compounds 4-7 could be found; therefore they were included in the testing.

Table IV. Cytotoxicity of the naphthoquinones.

\begin{tabular}{lccccccc}
\hline Cell line & $\begin{array}{c}\text { O-Phenethyl- } \\
\text { herbarin (1) }\end{array}$ & $\begin{array}{c}\text { Herbaridine } \\
\mathrm{A} \mathrm{(2)}\end{array}$ & $\begin{array}{c}\text { Herbaridine } \\
\mathrm{B} \mathrm{(3)}\end{array}$ & Herbarin (4) & $\begin{array}{c}\text { Dehydro- } \\
\text { herbarin (5) }\end{array}$ & $\begin{array}{c}O \text {-Methyl- } \\
\text { herbarin (6) }\end{array}$ & $\begin{array}{c}\text { Scorpinone } \\
\text { (7) }\end{array}$ \\
\hline Jurkat & 7.5 & 1.5 & 2 & 1 & 0.5 & 6 & 10 \\
Colo-320 & 2.5 & 2.5 & 0.5 & 0.5 & 0.5 & 2.5 & 5 \\
Neuro-2A & 15 & 5 & 2.5 & 2.5 & 2.5 & 7.5 & 20 \\
L-1210 & 10 & 2 & 0.5 & 2 & 2.5 & 7.5 & $>20$ \\
\hline
\end{tabular}


Table V. MICs of the naphthoquinones.

\begin{tabular}{lccccccc}
\hline Organism & \multicolumn{7}{c}{ MIC $[\mu \mathrm{g} / \mathrm{ml}]$} \\
& $\begin{array}{c}\text { O-Phene- } \\
\text { thyl-herbar- } \\
\text { in (1) }\end{array}$ & $\begin{array}{c}\text { Herbaridine } \\
\text { A (2) }\end{array}$ & $\begin{array}{c}\text { Herbaridine Herbarin (4) } \\
\text { B (3) }\end{array}$ & $\begin{array}{c}\text { Dehydro- } \\
\text { herbarin (5) }\end{array}$ & $\begin{array}{c}\text { O-Methyl- } \\
\text { herbarin (6) }\end{array}$ & $\begin{array}{c}\text { Scorpinone } \\
\text { (7) }\end{array}$ \\
\hline Micrococcus luteus & $>50$ & $>50$ & $>50$ & $>50$ & $>50$ & $>50$ & $>50$ \\
Bacillus brevis & $>50$ & $20 \mathrm{~s}$ & $20 \mathrm{~s}$ & $5 \mathrm{z}$ & $20 \mathrm{~s}$ & $20 \mathrm{z}$ & $5 \mathrm{z}$ \\
Bacillus subtilis & $>50$ & $20 \mathrm{~s}$ & $20 \mathrm{~s}$ & $5 \mathrm{z}$ & $20 \mathrm{~s}$ & $20 \mathrm{z}$ & $5 \mathrm{z}$ \\
Mycobacterium phlei & $>50$ & $20 \mathrm{~s}$ & $20 \mathrm{~s}$ & $5 \mathrm{~s}$ & $20 \mathrm{~s}$ & $20 \mathrm{~s}$ & $5 \mathrm{~s}$ \\
Enterobacter dissolvens & $>50$ & $>50$ & $>50$ & $>50$ & $>50$ & $>50$ & $>50$ \\
Escherichia coli & $>50$ & $20 \mathrm{~s}$ & $20 \mathrm{~s}$ & $5 \mathrm{~s}$ & $10 \mathrm{~s}$ & $20 \mathrm{~s}$ & $5 \mathrm{~s}$ \\
Nematospora coryli & $>50$ & $>50$ & $>50$ & 30 & 20 & 30 & $>50$ \\
\hline
\end{tabular}

s, Bacteriostatic; z, bactericidal.

The naphthoquinone derivatives showed moderate antibacterial activity except for $O$-phenethylherbarin (1) which showed no activity up to $50 \mu \mathrm{g} / \mathrm{ml}$. None of the tested filamentous fungi (Paecilomyces variotii, Penicillium notatum, Mucor miehei) was sensitive up to $50 \mu \mathrm{g} / \mathrm{ml}$ against compounds 1-7. Herbarin (4), dehydroherbarin (5) and $O$-methylherbarin (6) showed moderate activity against the yeast Nematospora coryli. Antimicrobial activity for a mixture of $\mathbf{4}$ and $\mathbf{5}$ could be found in the literature (Kadkol et al., 1971). For Bacillus subtilis and Micrococcus luteus, the MIC was 20 and $75 \mu \mathrm{g} / \mathrm{ml}$, respectively. For fungal species, the MIC exceeded $50 \mu \mathrm{g} / \mathrm{ml}$ except for Alternaria solani with $50 \mu \mathrm{g} / \mathrm{ml}$. Our findings correspond to the literature data with a MIC for $B a$ cillus subtilis of $5 \mu \mathrm{g} / \mathrm{ml}$ for $\mathbf{4}$ and $20 \mu \mathrm{g} / \mathrm{ml}$ for $\mathbf{5}$. The MICs for Micrococcus luteus and filamentous fungi exceeded $50 \mu \mathrm{g} / \mathrm{ml}$ which is consistent with the published data. The MIC values are listed in Table V.

Medentsev and Akimenko (1998) suggested that naphthoquinones are classical secondary metabolites of fungi and that their production is preferred under growth inhibition conditions. This was confirmed by the production of the metabolites of IBWF79B-90A eight days after complete consumption of glucose in the medium when the growth had already ceased. The structural relationship and the presumption of a common precursor of all the described compounds are obvious. The values for cytotoxicity, which ranged between 0.5 and $7.5 \mu \mathrm{g} / \mathrm{ml}$ for the closely related compounds herbaridine A (2), herbaridine B (3), herbarin (4), dehydroherbarin (5), and $O$-methylherbarin (6) were slightly higher than for $O$-phenethylherbarin (1) and scorpinone (7). For fusarubin and related compounds, the $\mathrm{IC}_{50}$ values for L-1210 cells range from 1.7 to $6.2 \mu \mathrm{g} /$ $\mathrm{ml}$ (Kurobane et al., 1986). This cytotoxicity of the fusarubin derivatives corresponds to those of the herbarin derivatives. The antimicrobial activity with values of 5 to $30 \mu \mathrm{g} / \mathrm{ml}$ against several bacteria and the yeast Nematospora coryli is modest. Herbarin (4) and scorpinone (7) showed the highest bioactivity with values of $5 \mu \mathrm{g} / \mathrm{ml}$ against the Bacillus species, Escherichia coli and Mycobacterium phlei. Kurobane et al. (1986) investigated fusarubin derivatives and the values of the MICs for Bacillus subtilis (12.5-50 $\mu \mathrm{g} / \mathrm{ml})$, Micrococcus luteus $(50-100 \mu \mathrm{g} / \mathrm{ml})$ and two different Candida albicans $(25-50 \mu \mathrm{g} / \mathrm{ml})$ agree with those of the structurally related herbarin derivatives. The impact of quinones like bostrycoidin and fusarubin on the respiratory chain was investigated by Bironaité et al. (1992) for bovine heart mitochondria and by Medentsev and Akimenko (1992) for pea seedlings - both suggested that the biological activity is caused by oxidative stress.

\section{Acknowledgement}

We thank Prof. Olov Sterner (University of Lund, Sweden) for the initial suggestion of the structures as well as Dr. Stephan Franke (University of Hamburg, Germany) for the mass spectrometric analyses. 
Anke H., Bergendorff O., and Sterner O. (1989), Assays of the biological activities of guaiane sesquiterpenoids isolated from the fruit bodies of edible $\mathrm{Lac}$ tarius species. Food Chem. Toxicol. 27, 393-397.

Arsenault G. P. (1965), The structure of bostrycoidin, a $\beta$-aza-anthraquinone from Fusarium solani $\mathrm{D}_{2}$ purple. Tetrahedron Lett. 45, 4033-4037.

Bironaité D. A., Čénas N. K., Anusevičius Ž. J., Medentsev A. G., Akimenko V. K., and Usanov S. A. (1992), Fungal quinone pigments as oxidizers and inhibitors of mitochondrial NADH:ubiquinone reductase. Arch. Biochem. Biophys. 297, 253-257.

Cameron D. W., Deutscher K. R., and Feutrill G. I. (1980), Synthesis of bostrycoidin and 8-O-methylbostrycoidin. Tetrahedron Lett. 21, 5089-5090.

Gottlieb H. E., Kotlyar V., and Nudelman A. (1997), NMR chemical shifts of common laboratory solvents as trace impurities. J. Org. Chem. 62, 7512-7515.

Hayashi T., Smith F. T., and Lee K.-H. (1987), Antitumor agents. 89. Psychorubin, a new cytotoxic naphthoquinone from Psychotria rubra and its structure-activity relationships. J. Med. Chem. 30, 2005-2008.

Kadkol M. V., Gopalkrishnan K. S., and Narasimhachari N. (1971), Isolation and characterization of naphthoquinone pigments from Torula herbarum (Pers.). Herbarin and dehydroherbarin. J. Antibiot. 4, 245-248.

Kesteleyn B. and De Kimpe N. (2000), Synthesis of two naphthoquinone antibiotics, dehydroherbarin and 6-deoxybostrycoidin. J. Org. Chem. 65, 640-644.

Kurobane I., Zaita N., and Fukuda A. (1986), New metabolites of Fusarium martii related to dihydrofusarubin. J. Antibiot. 39, 205-214.
Medentsev A. G. and Akimenko V. K. (1992), Mechanism of phytotoxic action of naphthoquinone pigments of the fungus Fusarium decemcellulare. Phytochemistry 31, 77-79.

Medentsev A. G. and Akimenko V. K. (1998), Naphthoquinone metabolites of the fungi. Phytochemistry 47, 935-959.

Miljkovic A., Mantle P. G., Williams D. J., and Rassing B. (2001), Scorpinone: a new natural azaanthraquinone produced by a Bispora-like tropical fungus. J. Nat. Prod. 64, 1251-1253.

Narasimhachari N. and Gopalkrishnan K. S. (1974), Naphthoquinone pigments from Torula herbarum: structure of $O$-methylherbarin. J. Antibiot. 27, 283-287.

Paranagama P. A., Wijeratne E. M. K., Burns A. M. Marron M. T., Gunatilaka M. K., Arnold A. E., and Gunatilaka A. A. L. (2007), Heptaketides from Corynespora sp. inhabiting the cavern beard lichen, Usnea cavernosa: first report of metabolites of an endolichenic fungus. J. Nat. Prod. 70, 1700-1705.

Schoettler S., Bascope M., Sterner O., and Anke T. (2006), Isolation and characterisation of two verrucarins from Myrothecium roridum. Z. Naturforsch. 61c, 309-314.

Tanaka H., Koyama Y., Nagai T., Marumo H., and Omura S. (1975), Nanaomycins, new antibiotics produced by a strain of Streptomyces. J. Antibiot. 28, 868-875. 